\title{
Two-dimensional Bose-Einstein condensate in an optical surface trap
}

\author{
D. Rychtarik, B. Engeser, H.-C. Nägerl, and R. Grimm \\ Institut für Experimentalphysik, Universität Innsbruck, Technikerstraße 25, A-6020 Innsbruck, Austria
}

(Dated: October 29, 2018)

\begin{abstract}
We report on the creation of a two-dimensional Bose-Einstein condensate of cesium atoms in a gravito-optical surface trap. The condensate is produced a few $\mu \mathrm{m}$ above a dielectric surface on an evanescent-wave atom mirror. After evaporative cooling by all-optical means, expansion measurements for the tightly confined vertical motion show energies well below the vibrational energy quantum. The presence of a condensate is observed in two independent ways by a magnetically induced collapse at negative scattering length and by measurements of the horizontal expansion.
\end{abstract}

PACS numbers: 32.80.Pj, 03.75.-b, 34.50.-s

Quantum gases in lower dimensions [1] currently attract considerable interest as model systems to study a wide range of phenomena related to statistical physics, condensed matter physics, and other areas. Dimensionally reduced systems may exhibit strikingly new properties in comparison to the three-dimensional case. BoseEinstein condensation (BEC) does not occur in 1D or 2D in the case of an infinite homogeneous system [2], but it is possible in highly anisotropic traps at finite particle number [3]. Only recently, experiments have entered regimes of BEC in $1 \mathrm{D}[4,5]$ or $2 \mathrm{D}[4]$.

The interest in 2D BEC goes back to early experiments on spin-polarized hydrogen on a liquid-helium surface [6], for which evidence of quantum degeneracy has been obtained 7]. The gradual onset of coherence and formation of quasi-condensates have already been discussed in this context [8]. Ultracold, laser-manipulated atomic quantum gases offer many more intriguing features like precise control of the external confinement, tunability of interatomic interactions, and superb experimental access. Novel phenomena like modified interaction properties have been predicted for $2 \mathrm{D}$ quantum gases tightly confined in one direction [9]. The exploration of these phenomena relies on the development of appropriate trapping schemes.

In this Letter, we report on the creation of a twodimensional BEC of cesium atoms by evaporative cooling in a highly anisotropic surface trap. Our gravito-optical surface trap (GOST) is based on an evanescent wave $(\mathrm{EW})$ atom mirror in combination with a horizontally confining optical dipole potential [10], see illustration in Fig. 11 The repulsive EW is formed on the surface of a prism by a blue-detuned laser beam undergoing total internal reflection. Since gravity pushes the atoms onto the prism the vertical motion is tightly confined in a gravitooptical potential

$$
U(z)=U_{0} \mathrm{e}^{-2 z / \Lambda}+m g z,
$$

where $z$ is the vertical distance from the surface and $U_{0}, m$, and $g$ denote the maximum EW potential, the atomic mass, and the gravitational acceleration, respectively. The EW field decay length is given by $\Lambda=$
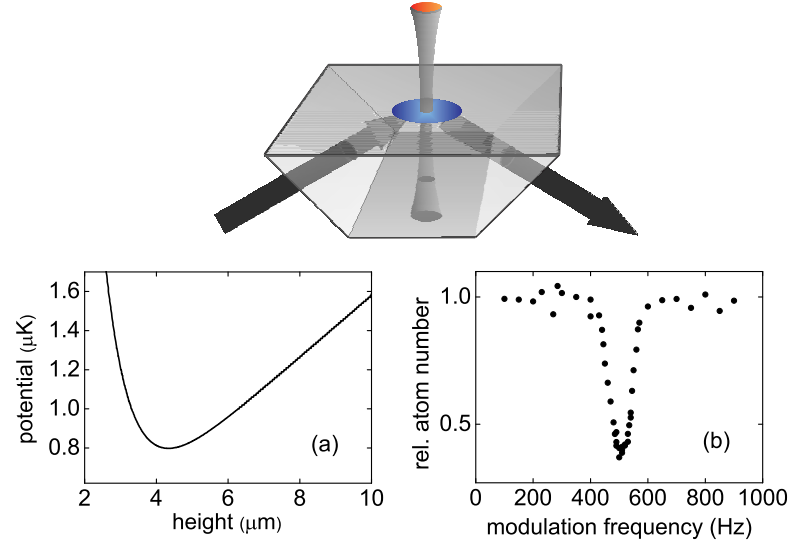

FIG. 1: Illustration of the gravito-optical surface trap together with (a) the calculated vertical potential and (b) a measurement of the vertical trap frequency.

$\lambda / 2 \pi \times\left(n^{2} \sin ^{2} \theta-1\right)^{-1 / 2}$, where $n$ is the refractive index of the medium. The potential minimum is located at a height $z_{0}=\frac{1}{2} \Lambda \ln \left(2 U_{0} / m g \Lambda\right)$ and provides a trap frequency of $\omega_{z}=(2 g / \Lambda)^{1 / 2}$. The quantized nature of the motion in such a trap and the behavior of a bosonic gas were theoretically investigated in [11, 12].

The trap loading procedure is based on methods described in our previous work [13, 14]. We release cesium atoms from a magneto-optical trap into a large-volume GOST where horizontal confinement is realized with a blue-detuned hollow beam (diameter $0.8 \mathrm{~mm}$, power $50 \mathrm{~mW}$, detuning $50 \mathrm{GHz}$ ). In this stage the EW is realized with a 100-mW diode laser and tuned a few $\mathrm{GHz}$ above resonance with atoms in the lower hyperfine state $(F=3)$. Evanescent-wave Sisyphus cooling provides a sample of $10^{7} \mathrm{Cs}$ atoms in $F=3$ at a temperature of $\sim 10 \mu \mathrm{K}$. Then we introduce the focussed, vertically propagating 5 - $\mathrm{W}$ laser beam of an $\mathrm{Yb}$ fibre laser (wavelength $1064 \mathrm{~nm}$, beam waist $130 \mu \mathrm{m}$ ) to provide a narrow dimple potential in the center of the trapped atom cloud with a depth of $\sim 50 \mu \mathrm{K}$ and a horizontal oscillation frequency of $130 \mathrm{~Hz}$. We wait for $1 \mathrm{~s}$ until the dimple is filled through elastic collisions with a high-density sample of unpolar- 
ized Cs atoms in $F=3$. Finally the blue-detuned hollow beam is turned off.

For further experiments it is crucial to strongly suppress photon scattering and light-induced loss. Therefore the near-resonant EW is replaced by a far-detuned EW, which is derived from the 700-mW beam of a Ti:Sapph laser at a wavelength of $839 \mathrm{~nm}$. This EW is produced in a nearly round spot on the surface of the fused-silica prism $(n=1.45)$ with a diameter of $500 \mu \mathrm{m}\left(1 / \mathrm{e}^{2}\right.$ intensity drop) and maximum potential height of $U_{0} \approx$ $k_{B} \times 50 \mu \mathrm{K}$. The angle of incidence $\theta$ is set $4.2(1.5) \mathrm{mrad}$ above the critical angle, which leads to calculated values of the decay length and the vertical trap frequency of $\Lambda=1.4(3) \mu \mathrm{m}$ and $\omega_{z} / 2 \pi=600(50) \mathrm{Hz}$. The corresponding gravito-optical potential is shown in Fig. 1(a). The potential minimum is located about $4 \mu \mathrm{m}$ above the surface. The trap frequency can be measured by parametric excitation. Fig. 1(b) shows a loss feature induced by modulating the EW power at the trap frequency yielding $\omega_{z} / 2 \pi=550(50) \mathrm{Hz}$ [15].

For evaporative cooling we optically pump the atoms into the lowest spin state $F=3, m_{F}=3$, which offers very favorable scattering properties. In this state two-body loss is completely suppressed and convenient magnetic tuning of the s-wave scattering length $a$ is possible over a wide range [16]. At a magnetic field of $26.8 \mathrm{G}$ where $a=440 a_{0}$ ( $a_{0}$ is Bohr's radius) we find an optimum situation with a large elastic cross section for evaporative cooling at moderate three-body loss 17, 18]. At this stage we have a sample of $10^{6}$ atoms at a temperature of $9 \mu \mathrm{K}$ with peak values for number and phasespace density of the order of $4 \times 10^{12} \mathrm{~cm}^{-3}$ and $5 \times 10^{-4}$, respectively.

Forced evaporative cooling is performed by ramping down the power of the red-detuned beam by more than three orders of magnitude. The total ramp consists of two subsequent exponential ramps. The first ramp reduces the power from initially $5 \mathrm{~W}$ down to $470 \mathrm{~mW}$ within $2 \mathrm{~s}$, the second ramp lowers the power further to a final ramp power $P_{\mathrm{f}}$ of typically a few $\mathrm{mW}$ in $3.5 \mathrm{~s}$. To counteract the density decrease when the potential is ramped down we adiabatically reduce the waist of the red-detuned beam to $64 \mu \mathrm{m}$ by moving the focussing lens synchronously with the 5.5-s ramp.

A release-and-recapture method 13, 14, 19] is applied to observe the vertical expansion of the sample and thus to measure the vertical expansion energy $E_{\text {exp }}$. We turn off the EW for a short variable release time. Without EW the sample drops onto the surface while undergoing a vertical expansion. Those atoms that hit the roomtemperature surface get lost. After the short release time the EW is turned on again to prevent the remaining atoms from hitting the surface, and their number is measured after recapture into the MOT by taking a fluorescence image. Corresponding measurements without release are used to normalize the data and to determine

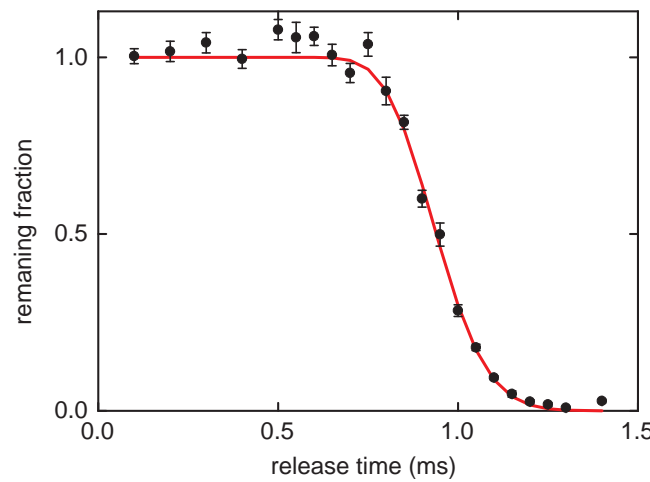

FIG. 2: Measurement of the vertical expansion energy with 2400 atoms left after deep evaporative cooling down to $P_{\mathrm{f}}=$ $1.6 \mathrm{~mW}$. The fraction of remaining atoms is plotted as a function of the release time. The fit of an error function to the data (solid lines) yields an expansion energy of $\frac{1}{2} k_{B} \times 16(2) \mathrm{nK}$ together with a release height of $4.3(1) \mu \mathrm{m}$.

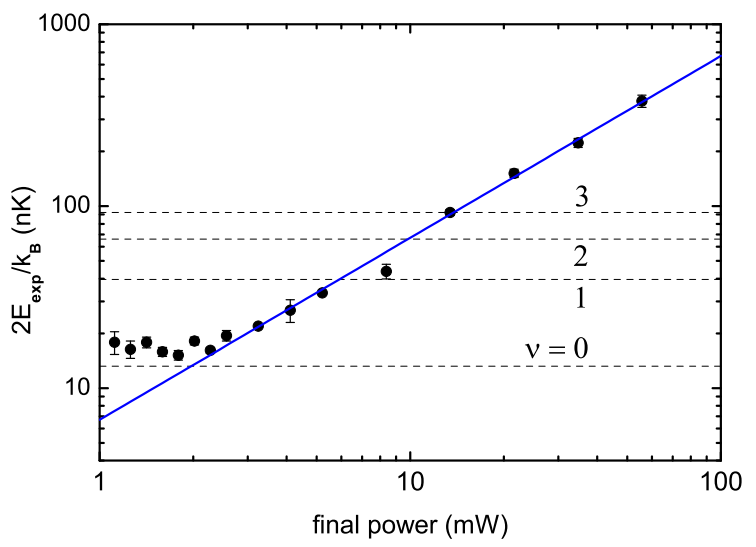

FIG. 3: Vertical expansion energy versus final power $P_{\mathrm{f}}$ of the evaporation ramp. The solid line illustrates the proportionality to the horizontal trap depth above $3 \mathrm{~mW}$. The dashed lines indicate the expansion energies for the four lowest vibrational levels.

the remaining fraction as a function of the release time. In the classical regime of a thermal gas with negligible effect of the quantized vertical motion, $E_{\exp }$ is directly related to the temperature $T$ by $E_{\exp }=\frac{1}{2} k_{B} T$, where $k_{B}$ is Boltzmann's constant. This classical regime ranges down to temperatures of $\sim 100 \mathrm{nK}$.

Fig. 2] shows a measurement taken with 2400 atoms remaining at $P_{\mathrm{f}}=1.6 \mathrm{~mW}$, where the horizontal trap frequency is $10 \mathrm{~Hz}$. We will show later that this sample is Bose condensed. The position of the sharp edge corresponds to the release height and the steepness is related to the energy spread. A corresponding fit of an error function to the data yields a release height of $z_{0}=4.3(1) \mu \mathrm{m}$ and an expansion energy $E_{\exp }=$ $\frac{1}{2} k_{B} \times 16(2) \mathrm{nK}$.

The measured vertical expansion energy is shown as a function of the final ramp power $P_{\mathrm{f}}$ in Fig. 3. With 
decreasing power down to about $3 \mathrm{~mW}$, we observe that $E_{\text {exp }}$ linearly follows the reduced horizontal trap depth $\hat{U}$ (see solid line). This shows that evaporative cooling takes place with an approximately fixed relation between temperature $T$ and trap depth $\hat{U}\left(k_{B} T / \hat{U} \approx 6\right)$. For power values below $3 \mathrm{~mW}$ we observe that $E_{\exp }$ levels off at about $\frac{1}{2} k_{B} \times 16 \mathrm{nK}$. This value is well below the vibrational energy quantum $\hbar \omega=k_{B} \times 26 \mathrm{nK}$ and close to the zero point energy of $k_{B} \times 13 \mathrm{nK}$. A comparison with the energies $E_{\nu}=\left(\nu+\frac{1}{2}\right) \hbar \omega_{z}$ of the quantum states in the vertical motion (see dashed lines in Fig. (3) shows that a two-dimensional gas is realized. This atomic "pancake" is created in a surface trap with an aspect ratio of about $50: 1$.

According to a calculation of the phase-space density based on the measured temperatures and the known trap frequencies, we expect the onset of BEC approximately at a final ramp power $P_{\mathrm{f}} \approx 10 \mathrm{~mW}$. Unfortunately, our release-and-recapture method does not provide sufficient information to extract two-component distributions in that regime and the data of Fig. 3. do not show any signature of a phase transition. We have therefore developed two other methods to show the presence of a BEC in the surface trap.

Our first method to prove BEC relies on a controlled collapse of the condensate at negative scattering length [20], a phenomenon that does not occur in a thermal gas. The scattering length is known to be negative for magnetic fields below $17 \mathrm{G}$ and the collapse of a Cs BEC below that field has been demonstrated in 17. In a series of collapse measurements performed with a trapped sample at $P_{\mathrm{f}}=1.6 \mathrm{~mW}$ we switched the magnetic field from the evaporation field of $26.8 \mathrm{G}$ to a variable field between $0 \mathrm{G}\left(a \approx-3000 a_{0}\right)$ and $40 \mathrm{G}\left(a \approx 1000 a_{0}\right)$ for a short time interval of $20 \mathrm{~ms}$ and measured the resulting loss. When the scattering length is negative the contraction of the BEC leads to a dramatic increase in density. In this case three-body recombination causes very fast loss. In a thermal gas, no contraction can happen and three-body loss at moderate scattering length is too slow to cause any significant reduction of the trapped atom number.

The magnetically induced collapse is demonstrated by the data in Fig. 4 For fields above the zero crossing at $17 \mathrm{G}$ switching at $a>0$ does not induce any significant loss. Below $17 \mathrm{G}$ where $a<0$ a rapid decrease in the trapped atom number is observed. Already at $a=-150 a_{0}$ realized at $14.7 \mathrm{G}$ almost half of the atoms are lost. This rapid loss at a field where the three-body loss coefficient is relatively small can only be explained by the huge density increase occurring during the condensate collapse.

In order to identify the final evaporation ramp power $P_{\mathrm{f}}$ where the phase transition occurs, we measure the induced collapse loss as a function of $P_{\mathrm{f}}$. In Fig. 国(a) we plot the fraction of atoms that is observed to remain after a 20 -ms application of a collapse field of $14.7 \mathrm{G}$. Above

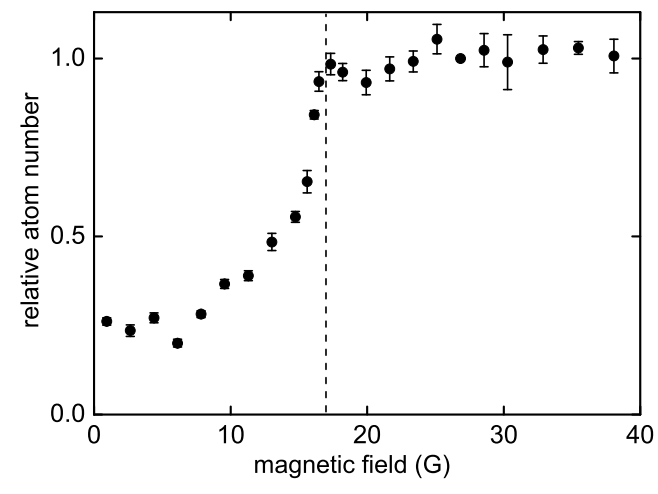

FIG. 4: Atom loss showing the magnetically induced BEC collapse at $P_{\mathrm{f}}=1.6 \mathrm{~mW}$. The magnetic field is switched to a variable value for a short time interval $20 \mathrm{~ms}$. The dashed line indicates the zero crossing of the scattering length below which the self-interaction is attractive.

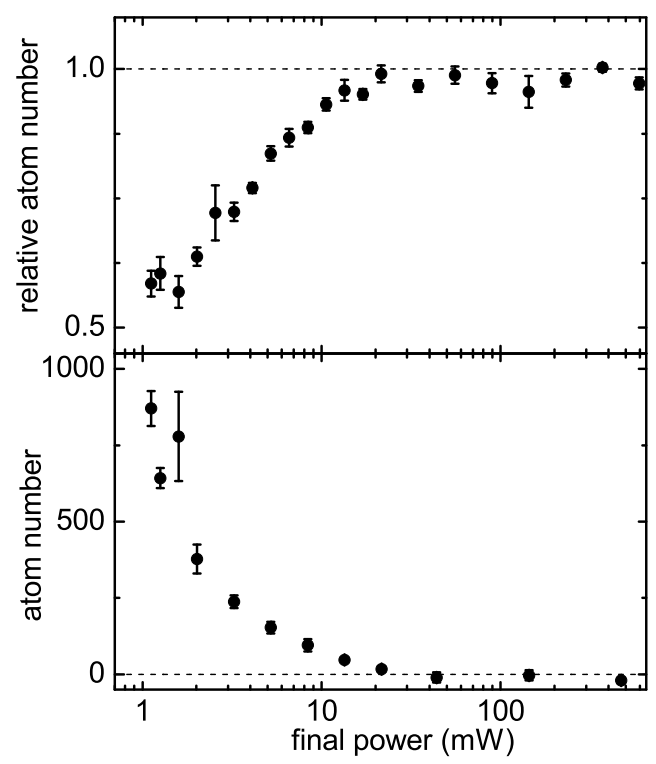

FIG. 5: Observation of the phase transition by two different methods. (a) The fraction of atoms remaining after a magnetically induced condensate collapse at $a=-150 a_{0}$ is plotted as a function of the final ramp power $P_{\mathrm{f}}$. (b) The horizontal release-and-recapture measurements show the remaining number of atoms after $400 \mathrm{~ms}$ of horizontal expansion.

$P_{\mathrm{f}} \approx 20 \mathrm{~mW}$ no significant loss is observed as expected for a thermal gas, which cannot undergo a collapse. Below $20 \mathrm{~mW}$ rapidly increasing loss sets in, which we interpret as the collapse of a growing condensate fraction. From this observation we infer that the phase transition takes place at a power of $P_{\mathrm{f}} \approx 20 \mathrm{~mW}$, where we have 15000 atoms left at a temperature of $150 \mathrm{nK}$. This is somewhat above the ramp power of $\sim 10 \mathrm{~mW}$ that we would expect from calculations of the phase-space density, but still lies in the corresponding error range.

Our second method to observe the onset of BEC is based on a horizontal release-and-recapture technique. 
After evaporation with a final ramp power $P_{\mathrm{f}}$ we turn off the horizontally confining attractive laser beam for a long time interval of $400 \mathrm{~ms}$. For recapturing the remaining atoms we then turn on the horizontally confining beam at a fixed power of $22 \mathrm{~mW}$. No atoms are recaptured for thermal samples $\left(P_{\mathrm{f}}>20 \mathrm{~mW}\right)$ as shown in Fig. [5 $\left.\mathrm{b}\right)$. For deeper evaporation with $P_{\mathrm{f}}$ below $20 \mathrm{~mW}$ we observe a rapidly increasing number of recaptured atoms which we interpret as a consequence of the much slower expansion of a condensate. The fact that the recapture signal occurs at the same final power as the onset of the collapse loss strongly supports this interpretation.

In the horizontal expansion measurements we observe significant recapture signals even after a few seconds of free evolution on the EW without the horizontally confining beam. This can not be explained by a slow expansion alone but rather indicates the presence of a horizontal trapping mechanism. An explanation might be the roughness of an EW atom mirror 21] leading to shallow potential wells. Surface imperfections of the prism or the interference with residual stray light can produce corrugations in the optical potential of the evanescent wave. A trapping effect could thus emerge from the pinning of the condensate to such defects.

With better knowledge on the phase transition we finally revisit the data of Fig. 3. The transition takes place at $P_{\mathrm{f}} \approx 20 \mathrm{~mW}$ in a $3 \mathrm{D}$ regime when the thermal component populates about five vertical quantum states. With decreasing values of $P_{\mathrm{f}}$ a crossover to a $2 \mathrm{D}$ regime takes place. For $P_{\mathrm{f}} \lesssim 2 \mathrm{~mW}$ a $2 \mathrm{D}$ BEC is formed, for which we estimate a chemical potential of $k_{B} \times 5 \mathrm{nK}$. In this $2 \mathrm{D}$ regime, the zero-point energy of $k_{B} \times 13 \mathrm{nK}$ dominates the vertical motion.

In order to further explore the properties of the $2 \mathrm{D}$ BEC in our GOST we are presently developing a new apparatus that offers much faster magnetic field control and much better optical access. These improvements will enable us to implement both in-situ and expansion imaging techniques. Magnetic levitation will allow us to overcompensate gravity and thus to observe a freely expanding atom cloud after release from the surface trap. For enhancing the anisotropy the two options are magnetic compression against the EW or the application of an additional attractive EW [19]. The trapped twodimensional condensate will allows us to study elementary excitations like solitons and vortices, the properties of which may exhibit striking differences as compared to the three-dimensional case. Further intriguing possibilities are offered by the creation of optical surface lattices created through the interference of different evanescent waves.

In conclusion, we have realized BEC in an optical surface trap based on an evanescent-wave atom mirror. During evaporative cooling we reach the phase transition in a 3D situation before the condensate is brought into the $2 \mathrm{D}$ regime by further increasing the trap anisotropy. The system opens up new possibilities to study the widely unexplored properties of degenerate quantum gases in two dimensions.

We gratefully acknowledge support by the Austrian Science Fund (FWF) within SFB 15 (project part 15) and by the European Community through the Research Training Network "FASTNet" under contract No. HPRN-CT-2002-00304.

[1] L. Pricoupenko, H. Perrin, and M. Olshanii, eds., Proceedings of the Euroschool on Quantum Gases in Lower Dimensions, Les Houches, 15 - 25 April 2003 (2003), J. Phys. IV, in press.

[2] V. Bagnato and D. Kleppner, Phys. Rev. A 44, 7439 (1991)

[3] W. Ketterle and N. J. van Druten, Phys. Rev. A 54, 656 (1996).

[4] A. Görlitz, J. Vogels, A. Leanhardt, C. Raman, T. Gustavson, J. Abo-Shaeer, A. Chikkatur, S. Gupta, S. Inouye, T. Rosenband, et al., Phys. Rev. Lett. 87, 130402 (2001).

[5] F. Schreck, L. Khaykovich, K. L. Corwin, G. Ferrari, T. Bourdel, J. Cubizolles, and C. Salomon, Phys. Rev. Lett. 87, 080403 (2001).

[6] J. T. M. Walraven, in Fundamental Systems in Quantum Optics, edited by J. Dalibard, J.M. Raimond, and J. Zinn-Justin (Elsevier, Amsterdam, 1992), p. 485.

[7] A. I. Safonov, S. A. Vasilyev, I. S. Yasnikov, I. I. Lukashevich, and S. Jaakkola, Phys. Rev. Lett. 81, 4545 (1998).

[8] Y. Kagan, B. Svistunov, and G. Shlyapnikov, Sov. Phys. JETP 66, 314 (1987).

[9] D. Petrov, M. Holzmann, and G. Shlyapnikov, Phys. Rev. Lett. 84, 2551 (2000).

[10] R. Grimm, M. Weidemüller, and Y. Ovchinnikov, Adv. At. Mol. Opt. Phys. 42, 95 (2000).

[11] H. Wallis, J. Dalibard, and C. Cohen-Tannoudji, Appl. Phys. B 54, 407 (1992).

[12] H. Wallis, Quantum Semiclass. Opt. 8, 727 (1996).

[13] Y. B. Ovchinnikov, I. Manek, and R. Grimm, Phys. Rev. Lett. 79, 2225 (1997).

[14] M. Hammes, D. Rychtarik, H.-C. Nägerl, and R. Grimm, Phys. Rev. A 66, 051401(R) (2002).

[15] The loss maximum occurs somewhat below the trap frequency because of the anharmonicity of the potential.

[16] A. J. Kerman, C. Chin, V. Vuletić, S. Chu, P. J. Leo, C. J. Williams, and P. S. Julienne, C. R. Acad. Sci. Paris IV 2, 633 (2001).

[17] T. Weber, J. Herbig, M. Mark, H.-C. Nägerl, and R. Grimm, Science 299, 232 (2003).

[18] T. Weber, J. Herbig, M. Mark, H.-C. Nägerl, and R. Grimm, Phys. Rev. Lett. 91, 123201 (2003).

[19] M. Hammes, D. Rychtarik, B. Engeser, H.-C. Nägerl, and R. Grimm, Phys. Rev. Lett. 90, 173001 (2003).

[20] J. L. Roberts, N. R. Clausen, S. L. Cornish, E. A. Donley, E. A. Cornell, and C. E. Wieman, Phys. Rev. Lett. 86, 4211 (2001).

[21] C. Henkel, K. Molmer, R. Kaiser, N. Vansteenkiste, C. I. Westbrook, and A. Aspect, Phys. Rev. A 55, 1160 (1997). 